\title{
Prognostic Significance of Preoperative Serum Alpha- fetoprotein in Hepatocellular Carcinoma and Correlation with Clinicopathological Factors: a Single-center Experience from China
}

\author{
Song-Lin An ${ }^{1}$, Ting Xiao², Li-Ming Wang ${ }^{1}$, Wei-Qi Rong ${ }^{1}$, Fan Wu ${ }^{1}$, Li Feng ${ }^{1}$, \\ Fa-Qiang Liu ${ }^{1}$, Fei Tian ${ }^{1}$, Jian-Xiong $\mathrm{Wu}^{1 *}$
}

\begin{abstract}
Objectives: To investigate the prognosis significance of preoperative serum alpha-fetoprotein (AFP) and the correlation with clinicopathological factors of hepatocellular carcinoma (HCC) patients who underwent hepatectomy. Materials and Methods: Clinicopathological data of retrospective analysis were collected for 251 HCC patients undergoing hepatectomy in this study. According to preoperative AFP level, patients were categorized into AFP-negative $(0-20 \mathrm{ng} / \mathrm{mL})$ and AFP-positive $(>20 \mathrm{ng} / \mathrm{mL})$ groups for Kaplan-Meier analysis and Cox proportional hazard regression modeling. Results: The results demonstrated that increased AFP was associated with longer prothrombin time (PTs), liver capsule invasion, low grade differentiation, and late Barcelona Clinic Liver Center (BCLC) stage. Moreover, the female patients had a greater prevalence of increased preoperative AFP than male patients [284.8 (3.975-3167.5) vs $(3.653-140.65)$; Z-2.895, $p=0.004]$. The 1-, 3-, and 5-year recurrence-free survival (RFS) rates were 78.1, 57.5, and 40.6 \% in the AFP-negative group and 61.8, 37.7, and 31.4\%, respectively, in the AFP-positive group (log-rank test 8.312, $p=0.004$ ). The 1-, 3-, and 5-year overall survival (OS) rates were 94.4, 83.8, and 62.3\% in the AFP-negative group and 87.2, 60.0, and 36.7\%, respectively, in the AFP-positive group. The difference was statistically significant (log-rank test, 16.884, $p=0.000$ ). Cox proportional-hazards model identified preoperative AFP to be an independent prognostic predictor of overall survival. Conclusions: Preoperative serum AFP is an independent predictor of prognosis among HCC patients following surgical resection. Female patients have a higher preoperative AFP than their male counterparts.
\end{abstract}

Keywords: Hepatocellular carcinoma - alpha-fetoprotein - hepatectomy - survival

Asian Pac J Cancer Prev, 16 (10), 4421-4427

\section{Introduction}

Hepatocellular carcinoma (HCC) is the sixth most common cancer worldwide and the third most frequent cause of cancer-related death. Half of these cases and deaths are estimated to occur in China (Ferlay et al., 2010; Jemal et al., 2011). Although there is substantial geographical variation, the greatest burden of the disease is in East Asia, Eastern Europe and sub-Saharan Africa, where hepatitis B virus (HBV) infection is highly prevalent (Yuen et al., 2009). HBV infection is the predominant cause of HCC development, associated with approximately half of all cases of $\mathrm{HCC}$, and almost all cases of HCC in children (El-Serag et al., 2011). By contrast, in North America, Europe, and Japan, infection with hepatitis $\mathrm{C}$ virus (HCV) is the main risk factor. In addition to $\mathrm{HBV}$ and $\mathrm{HCV}$ infections, increasing age, male sex and chronic alcohol consumption are significant risk factors for the development of HCC. HCC incidence rates are increasing in many parts of the world including the United States and Central Europe, possibly due to these factors (Bosetti et al., 2008; Center et al., 2011).

Alpha-fetoprotein (AFP), a fetal specific glycoprotein, is synthesized in the liver of human embryos as early as 1 month after conception and is present in greatest quantities around the $3^{\text {rd }}$ month of gestation, and its synthesis will cease at or near birth, and then the concentration will decline to a low level (Tomasi et al., 1977). In healthy adults, serum AFP concentration is below 20ng/ml. AFP has served as a useful biomarker for diagnosis of HCC since the 1970s, when most patients with HCC were diagnosed at an advanced stage and had clinical symptoms (Debruyne et al., 2008). Currently, liver ultrasonography (US) combined with AFP measurement every 6 months is the standard method of HCC surveillance in many Countries and regions (Kim et al., 2012).

${ }^{1}$ Department of Abdominal Surgical Oncology, ${ }^{2}$ Department of Etiology and Carcinogenesis, State Key Laboratory of Molecular Oncology, Cancer Institute and Hospital, Peking Union Medical College and Chinese Academy of Medical Sciences, Beijing, China *For correspondence:dr.wujx@hotmail.com 
The prognosis value of AFP has been proposed in recent studies, although the clinical utility of AFP for HCC remains controversial. Some studies found that elevated serum AFP levels are a robust predictor of poor overall survival and recurrence-free survival (Chan et al., 2009; Lee et al., 2012). While, other researchers found that serum AFP is not a good prognostic indicator in small HCC, especially in HCC $\leq 2 \mathrm{~cm}$ (Forner et al., 2008; Giannini et al., 2012).

Therefore, in this study, we collected and analyzed the clinical and follow-up information of $\mathrm{HCC}$ underwent hepatectomy to evaluate the prognostic role of AFP in Chinese patients. Moreover, we expect to reveal the differences of clinicopathological factors between the AFP-negative patients and the AFP-positive ones.

\section{Materials and Methods}

\section{Patients}

Between January 2006 and December 2011, two hundreds and fifty-one consecutive HCC patients (27 to 79 years of age) who underwent hepatectomy at the Cancer Institute and Hospital of Chinese Academy of Medical Science were enrollment in the study. The diagnostic criteria for HCC used in the study were in accordance with the American Association for the Study of Liver Diseases' 2005 guidelines. All patients had preoperative serum AFP levels $>200 \mathrm{ng} / \mathrm{ml}$ or a typical enhancement pattern (arterial enhancement and portal/delayed washed out) on dynamic imaging of hepatic mass (es) $>2 \mathrm{~cm}$, or cytologic/histologic evidence of HCC

Preoperative procedures consisted of blood routine, liver and renal function, coagulation studies, screening for $\mathrm{HBV} / \mathrm{HCV}$ markers, and determination of serum AFP, carcinoembryonic antigen (CEA), and carbohydrate antigen 199 (CA199)levels. Abdominal computed tomography scan with contrast or magnetic resonance imaging was routinely undertaken. Child-Pugh criteria were used for liver function evaluation. Tumor staging was evaluated according to the Barcelona Clinic Liver Cancer (BCLC) staging classification. All patients provided written informed consent before surgery and the treatments were performed in accordance with present ethical principles.

\section{Sample classification}

As the cut-off value for AFP is set at $20 \mathrm{ng} / \mathrm{ml}$ in most studies (Debruyne Gupta et al., 2003; Zhang et al., 2010), the 251 cases were classified into two groups using this cut-off value; therefore, 142 cases were classified in the AFP-negative group (AFP $\leq 20 \mathrm{ng} / \mathrm{ml})$, and 109 cases were classified in the AFP-positive group (AFP>20ng/ml).

\section{Follow-up}

All postoperative patients were followed at 3-monthly intervals for the first year and at 4- to 6-monthly intervals thereafter. The follow-up program included serum AFP assays, liver function tests, abdominal ultrasonography, and chest X-rays. Enhanced computed tomography or magnetic resonance imaging was performed every 6 months for surveillance of recurrence. Bone scanning was undertaken when necessary. In cases where a suspicious recurrent or metastatic lesion was detected, magnetic resonance imaging or hepatic angiography was employed to consolidate the diagnosis. The diagnosis of tumor recurrence was based on typical imaging features, an increase in serum AFP levels or development of extrahepatic metastasis. Fine needle aspiration/biopsies were not necessarily undertaken to assess recurrences.

\section{Statistical analysis}

Statistical analysis performed using SPSS version 17.0 software. Continuous variables of normal distribution were expressed as mean and SD and compared with the independent T-test. Continuous variables of non-normal distribution were expressed as medians and IQRs and compared with the Mann-Whitney U test. Categorical variables were compared by the chi-square test or Fisher's exact test as appropriate. Overall survival (OS) and recurrence-free survival (RFS) rates were evaluated by Kaplan-Meier survival estimates test, and the $\mathrm{P}$ values were assessed using log-rank tests. For multivariate analysis, the stepwise Cox's proportional-hazards models were used. In all cases, statistical significance was defined as $p<0.05$.

\section{Results}

\section{Patient characteristics}

The demographic, clinical, and pathologic characteristics of the 251 patients are shown in Table 1 . There were 214 men and 37 women, with a mean age of 55 years (range 27-79). A total of $90.84 \%$ (228/251) of patients had hepatitis B or C virus infection and $86.45 \%$ (217/251) had liver cirrhosis of varying severity.

Comparison of the AFP-negative and AFP-positive groups In our study, AFP displayed a sensitivity of $52.2 \%$; the characteristics of the two groups are shown in Table 1. The AFP-positive group exhibited characteristics of poor tumor biological behavior (liver capsule invasion, and low grade differentiation), poor liver function (abnormal prothrombin time), and late BCLC stage.

Moreover, the female ratio in the AFP-positive group was higher than that in the AFP-negative group, and the difference was significant (24/109 vs 13/124, $p=0.004)$. And, the female patients had a greater prevalence of increased serum preoperative AFP than male patients [284.8 (3.975-3167.5) vs (3.653-140.65); $\mathrm{Z}=-2.895$, $p=0.004]$.

\section{Operative variables and perioperative outcomes}

Operative and perioperative variables are summarized in Table 2. Total intra-operative blood loss and blood transfusion rates in the AFP-positive group were greater than in the AFP-negative group. In 72 patients with an uncomplicated procedure, hepatectomy was performed without blood occlusion. A selective and dynamic regionspecific vascular occlusion (SDRVO) technique (Yu et al., 2014) was used in 153 patients. Pringle was performed during the operation in 26 patients. On postoperative day 3, the level of PTs in the AFP-positive group was 
Table 1. Clinical and Pathologic Characteristics

\begin{tabular}{|c|c|c|c|}
\hline Variables & AFP-negative group $(n=142)$ & AFP-positive group $(n=109)$ & $P$ value \\
\hline Age(years) & $55.23 \pm 11.375$ & $53.16 \pm 10.112$ & 0.129 \\
\hline Sex & & & 0.004 \\
\hline male & 129 & 85 & \\
\hline female & 13 & 24 & \\
\hline Cirrhotic liver (yes/no) & $120 / 22$ & Dec-97 & 0.304 \\
\hline Chronic hepatitis & & & 0.091 \\
\hline No & 18 & 5 & \\
\hline $\mathrm{HBV}$ & 113 & 97 & \\
\hline $\mathrm{HCV}$ & 9 & 4 & \\
\hline $\mathrm{HBV}+\mathrm{HCV}$ & 2 & 3 & \\
\hline Alcohol intake (yes/no) & $83 / 59$ & $75 / 34$ & 0.092 \\
\hline $\operatorname{ALT}(\mathrm{U} / \mathrm{L})$ & $34.00(24.75-55.00)$ & $32.00(21.00-49.00)$ & 0.079 \\
\hline GGT (U/L) & $52.00(30.75-79.25)$ & $53.00(32.00-91.00)$ & 0.432 \\
\hline TBIL (umol/L) & $11.30 \quad(8.45-15.80)$ & $12.45(8.98-16.15)$ & 0.358 \\
\hline $\operatorname{ALB}(g / L)$ & $41.18 \pm 4.013$ & $40.95 \pm 4.538$ & 0.678 \\
\hline PLT & $150.58 \pm 48.948$ & $146.78 \pm 52.848$ & 0.558 \\
\hline PTs & $11.95(11.48-12.50)$ & $12.15(11.58-12.80)$ & 0.038 \\
\hline BCLC staging & & & 0.025 \\
\hline stage A & 92 & 56 & \\
\hline stage $\mathrm{B}$ & 45 & 43 & \\
\hline stage $\mathrm{C}$ & 4 & 10 & \\
\hline \multicolumn{4}{|l|}{ Number of tumor } \\
\hline 1 & 126 & 96 & 0.871 \\
\hline$\geq 2$ & 16 & 13 & \\
\hline Tumor diameter $(\mathrm{cm})$ mean $\pm \mathrm{SD}$ & $4.87 \pm 2.778$ & $5.54 \pm 3.494$ & 0.091 \\
\hline Blood vessel invasion (yes/no) & $11 / 131$ & $14 / 95$ & 0.181 \\
\hline Liver capsule invasion (yes/no) & $80 / 62$ & $77 / 32$ & 0.02 \\
\hline Differentiation & & & 0 \\
\hline Well & 26 & 2 & \\
\hline Moderate & 93 & 70 & \\
\hline Poor & 23 & 37 & \\
\hline incision margin & & & 0.554 \\
\hline$\leq 1.0 \mathrm{~cm}$ & 65 & 54 & \\
\hline$>1.0 \mathrm{~cm}$ & 77 & 55 & \\
\hline Preoperative TACE (yes/no) & $129 / 13$ & $93 / 16$ & 0.175 \\
\hline
\end{tabular}

*Continuous variables of normal distribution were expressed as mean and SD and compared with the independent T-test. Continuous variables of non-normal distribution were expressed as medians and IQRs and compared with the Mann-Whitney U test. Categorical variables were compared by the chi-square test or Fisher's exact test as appropriate; BCLC Barcelona Clinic Liver Cancer, HBV hepatitis B virus, HCV hepatitisC virus, IQR interquartile SD standard deviation, TACE transarterial chemoembolization

significantly higher than in the AFP-negative group, but there was no significant difference between the two groups on postoperative day 7. Transient liver impairment was defined as Child's C status on postoperative day 7. The number of cases with post-operative massive hemorrhage, bile leakage, transient liver impairment, and 30-day operative mortality was $1,5,13$, and 0 , respectively, with no significant difference between the two groups.

\section{Recurrence}

$\mathrm{HCC}$ recurrence ratio in the AFP-positive group was higher than that in the AFP-negative group [69/109 (63.30\%) vs 67/142 (47.18\%); $p=0.011]$. Most recurrence $(116 / 136)$ developed in liver and there were no differences between the two groups for the site or number of recurrence (Table 3). Most recurrence (110/136) occurred in 2 years after hepatectomy and the ratio in the AFP-positive group was higher than that in the AFP-negative group [61/69(88.41\%) vs 49/67 (73.13\%); $p=0.024]$. TACE was received by 70 patients as the main initial therapy for $\mathrm{HCC}$ recurrence. A total of 32 patients received potentially curative treatment for recurrence of HCC, including RFA in 24 patients and surgery in 8 patients.

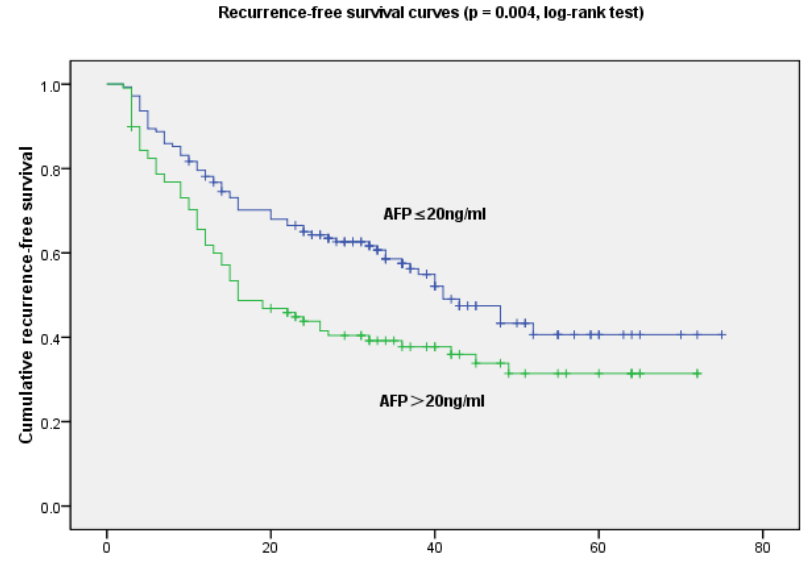

Figure 1. Recurrence-free Survival Time (Months)

The 1-, 3-, and 5-year recurrence-free survival rates for all patients were $77.1,49.0$, and $36.8 \%$, respectively. The rates were $78.1,57.5$, and $40.6 \%$ in the AFP-negative group and $61.8,37.7$, and $31.4 \%$, respectively, in the AFP-positive group (Figure 1). The differences were statistically significant in favor of the AFP-negative group (log-rank test 8.312, $p=0.004$ ). 
Table 2. Operative Variables and Peri-operative Outcomes

\begin{tabular}{|c|c|c|c|c|c|}
\hline \multirow{2}{*}{$\frac{\text { Variables }}{\text { Intraoperative variables }}$} & \multicolumn{2}{|c|}{ AFP-negative group $(n=142)$} & \multicolumn{2}{|c|}{ AFP-positive group $(n=109)$} & \multirow[t]{2}{*}{$P$ value } \\
\hline & \multirow{3}{*}{\multicolumn{2}{|c|}{$195(155-240)$}} & \multirow{3}{*}{\multicolumn{2}{|c|}{$210(162.5-297.5)$}} & \\
\hline Operative time (min) & & & & & 0.072 \\
\hline Vascular occlusion & & & & & 0.639 \\
\hline no & & 38 & & 34 & \\
\hline $\mathrm{SDRVO}^{\mathrm{a}}$ & & 90 & & 63 & \\
\hline Pringle & & 14 & & 12 & \\
\hline Warm ischemia time $(\min )^{b}$ & & $18(10-15)$ & & $17(0-25)$ & 0.354 \\
\hline Total blood loss (ml) & & $300(200-600)$ & & $500(300-1200)$ & 0.005 \\
\hline Blood transfusion (yes/no) & & $17 / 125$ & & $34 / 75$ & 0 \\
\hline Postoperative outcome & & 2 & & 3 & \\
\hline Alanine aminotransferase (U/L) 3 days & 201.50 & $129.75-298.25)$ & 241.00 & $(121.00-429.00)$ & 0.108 \\
\hline$\gamma$ glutamyltranspeptidase (U/L) 3 days & 45.00 & $(25.75-76.00)$ & 41.00 & $(30.25-64.00)$ & 0.722 \\
\hline Total bilirubin (mmol/L) 3 days & 18.90 & $(14.38-25.83)$ & 18.80 & $(13.53-26.63)$ & 0.936 \\
\hline Serum albumin $(\mathrm{g} / \mathrm{L}) 3$ days & 28.03 & $(30.80-32.55)$ & 27.65 & $(30.50-33.88)$ & 0.781 \\
\hline PTs 3 days & 12.45 & $(12.18-13.50)$ & 12.56 & $(12.19-13.80)$ & 0.041 \\
\hline Alanine aminotransferase (U/L) 7 days & 67.50 & $(47.00-98.50)$ & 90.00 & $(59.00-134.50)$ & 0.168 \\
\hline$\gamma$ glutamyltranspeptidase (U/L) 7 days & 85.00 & $(44.5-164.25)$ & 76.50 & $(61.50-128.50)$ & 0.957 \\
\hline Total bilirubin (mmol/L) 7 days & 16.4 & $(11.33-21.15)$ & 18.35 & $(10.88-30.55)$ & 0.41 \\
\hline Serum albumin $(\mathrm{g} / \mathrm{L}) 7$ days & 32.0 & $(30.03-34.25)$ & 32.55 & $(30.58-35.90)$ & 0.246 \\
\hline PTs 7 days & 12.16 & $(11.56-12.93)$ & 12.17 & $(11.59-12.96)$ & 0.062 \\
\hline Massive hemorrhage & & 0 & & 1 & 1 \\
\hline Bile leakage & & 3 & & 2 & 1 \\
\hline Transient liver impairment ${ }^{\mathrm{c}}$ & & 7 & & 6 & 0.839 \\
\hline 30-day operative mortality & & 0 & & 0 & 1 \\
\hline Postoperative hospital stay (day) & & $9(7-12)$ & & $9(7-13)$ & 0.77 \\
\hline
\end{tabular}

*Continuous variables of normal distribution were expressed as mean and SD and compared with the independent T-test. Continuous variables of non-normal distribution were expressed as medians and IQRs and compared with the Mann-Whitney U test. Categorical variables were compared by the chi-square test or Fisher's exact test as appropriate; a selective and dynamic region-specific vascular occlusion; ${ }^{\text {b} T o t a l ~ i s c h e m i a ~ t i m e ~ f o r ~ s e l e c t i v e ~}$ and dynamic region-specific vascular occlusion; 'Child's $\mathrm{C}$ status on postoperative day 7

Table 3. Characteristics and Treatment of Recurrent HCC

\begin{tabular}{lccc}
\hline Variables & $\begin{array}{c}\text { AFP-negative } \\
\text { group } \\
(\mathrm{n}=142)\end{array}$ & $\begin{array}{c}\text { AFP-positive } \\
\text { group } \\
(\mathrm{n}=109)\end{array}$ & P value \\
\hline Number of recurrence & 67 & 69 & 0.011 \\
\hline site of recurrence & & & 0.943 \\
$\quad$ IHR & 57 & 59 & \\
$\quad$ EHR & 10 & 10 & \\
recurrence time & & & 0.024 \\
in 2 years & 49 & 61 & \\
$\quad$ 2 years & 18 & 8 & 0.892 \\
treatment for recurrence & & & \\
$\quad$ TACE & 35 & 35 & \\
RFA & 11 & 13 & \\
$\quad$ Surgery & 5 & 3 & \\
$\quad$ Radiotherapy & 0 & 1 & \\
$\quad$ Sorafenib & 2 & 2 & \\
$\quad$ Supportive treatment & 14 & 15 & \\
\hline
\end{tabular}

*EHR extrahepatic recurrence, HCC hepatocellular carcinoma, IHR intrahepatic recurrence, RFA radiofrequency ablation, TACE transarterial chemoembolization; Variables were compared by the chi-square test or Fisher exact test as appropriate. The first recurrences that were recorded; ${ }^{a}$ The initial treatment for first recurrence

\section{Survival}

The median follow-up was 44(3-75) months. There were $85(33.86 \%)$ deaths at the time of data censor. In the AFP-negative group, 33 patients died as a result of tumor progression, in the AFP-positive group, the causes of death were tumor progression (52 patients) and liver failure (one patient). The 1-, 3-, and 5-year overall survival rates for

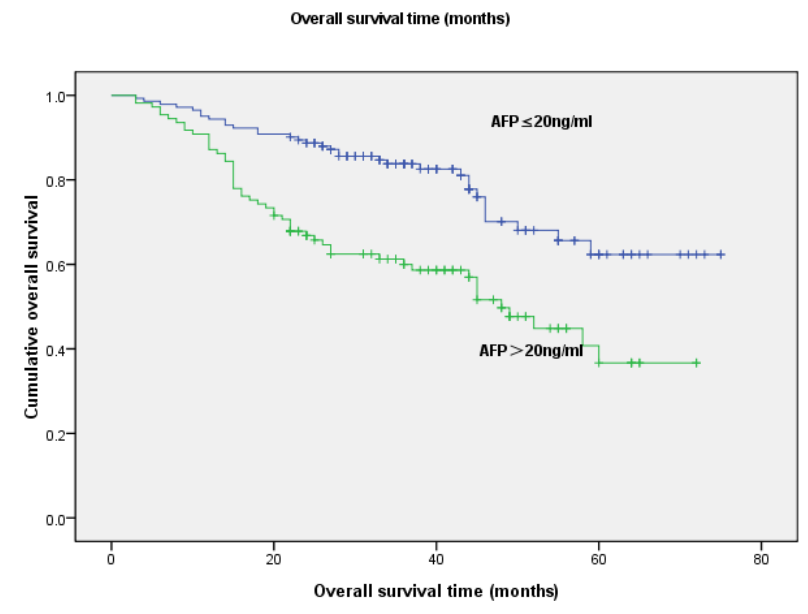

Figure 2. Overrall Survival Time (Months)

all patients were $91.2,73.5$, and $50.9 \%$ respectively. The rates were $94.4,83.8$, and $62.3 \%$ in the AFP-negative group and 87.2, 60.0, and $36.7 \%$, respectively, in the AFPpositive group (Figure 2). The difference was statistically significant (log-rank test 16.884, $p=0.000$ ).

\section{Prognostic factors}

We performed an analysis of prognostic factors for recurrence and survival. Exploratory multivariate analysis using a stepwise Cox proportional-hazards model identified three characteristics as independent prognostic predictors of recurrence (Table 4). These were tumor diameter (HR 1.158; 95\%CI 1.067-1.256; $p=0.000$ ), blood vessel invasion (HR 1.677; 95\%CI 1.009-2.787; 
Prognostic Significance of Preoperative Serum Alpha-fetoprotein in Hepatocellular Carcinoma in China Table 4. Univariate and Multivariate Analysis of Predictive Factors for Recurrence Free Survival

\begin{tabular}{|c|c|c|c|c|}
\hline \multirow[t]{2}{*}{ Variables } & \multicolumn{2}{|c|}{ Univariate analysis } & \multicolumn{2}{|c|}{ Multivariate analysis } \\
\hline & $\operatorname{HR}(95.0 \% \mathrm{CI})$ & $p$ value & $\mathrm{HR}(95.0 \% \mathrm{CI})$ & $p$ value \\
\hline Age & $1.010(0.994-1.026)$ & 0.225 & & \\
\hline Sex & $1.011(0.622-1.624)$ & 0.965 & & \\
\hline Chronic hepatitis & $0.924(0.658-1.298)$ & 0.648 & & \\
\hline Alcohol intake & $1.025(0.725-1.450)$ & 0.888 & & \\
\hline Cirrhotic liver & $1.414(0.813-2.460)$ & 0.22 & & \\
\hline AFP & $1.626(1.259-2.280)$ & 0.005 & $1.325(0.921-1.907)$ & 0.129 \\
\hline ALT & $0.999(0.994-1.004)$ & 0.763 & & \\
\hline GGT & $1.001(0.999-1.003)$ & 0.321 & & \\
\hline TBIL & $1.004(0.995-1.014)$ & 0.39 & & \\
\hline ALB & $0.972(0.933-1.012)$ & 0.163 & & \\
\hline PLT & $0.999(0.996-1.003)$ & 0.602 & & \\
\hline PTs & $1.077(0.884-1.312)$ & 0.463 & & \\
\hline Number of tumor & $1.609(1.021-2.537)$ & 0.04 & $1.393(0.858-2.261)$ & 0.18 \\
\hline Tumor diameter & $1.170(1.113-1.230)$ & 0 & $1.158(1.067-1.256)$ & 0 \\
\hline Liver capsule invasion & $2.010(1.380-2.927)$ & 0 & $1.318(0.874-1.988)$ & 0.188 \\
\hline Blood vessel invasion & $2.285(1.403-3.721)$ & 0.001 & $1.677(1.009-2.787)$ & 0.046 \\
\hline $\begin{array}{l}\text { Differentiation } \\
\text { D }\end{array}$ & $1.770(1.316-2.381)$ & 0 & $1.487(1.075-2.059)$ & 0.017 \\
\hline BCLC staging & $1.775(1.356-2.325)$ & 0 & $0.840(0.541-1.305)$ & 0.438 \\
\hline incision margin & $1.066(0.760-1.496)$ & 0.712 & & \\
\hline Operative time & $1.001(0.999-1.003)$ & 0.521 & & \\
\hline Warm ischemia time & $0.995(0.980-1.011)$ & 0.552 & & \\
\hline Blood transfusion & $1.577(1.068-2.327)$ & 0.022 & $1.090(0.696-1.707)$ & 0.708 \\
\hline Preoperative TACE & $1.478(0.887-2.463)$ & 0.113 & & \\
\hline
\end{tabular}

Table 5. Univariate and Multivariate Analysis of Predictive Factors for Overall Survival

\begin{tabular}{|c|c|c|c|c|}
\hline \multirow[t]{2}{*}{ Variables } & \multicolumn{2}{|c|}{ Univariate analysis } & \multicolumn{2}{|c|}{ Multivariate analysis } \\
\hline & $\operatorname{HR}(95.0 \% \mathrm{CI})$ & $p$ value & $\mathrm{HR}(95.0 \% \mathrm{CI})$ & $p$ value \\
\hline Age & $1.011(0.992-1.031)$ & 0.248 & & \\
\hline Sex & $1.091(0.604-1.970)$ & 0.773 & & \\
\hline Chronic hepatitis & $0.886(0.583-1.349)$ & 0.574 & & \\
\hline Alcohol intake & $0.791(0.503-1.243)$ & 0.309 & & \\
\hline Cirrhotic liver & $1.721(0.794-3.731)$ & 0.169 & & \\
\hline $\mathrm{AFP}$ & $2.417(1.561-3.743)$ & 0 & $1.767(1.108-2.816)$ & 0.017 \\
\hline ALT & $0.995(0.986-1.003)$ & 0.233 & & \\
\hline GGT & $1.002(0.999-1.004)$ & 0.149 & & \\
\hline TBIL & $1.009(1.000-1.018)$ & 0.047 & $1.675(0.903-3.106)$ & 0.102 \\
\hline ALB & $0.969(0.919-1.021)$ & 0.242 & & \\
\hline PLT & $0.997(0.992-1.001)$ & 0.171 & & \\
\hline PTs & $1.038(0.808-1.333)$ & 0.77 & & \\
\hline Number of tumor & $2.313(1.408-3.800)$ & 0.001 & $1.857(1.071-3.221)$ & 0.028 \\
\hline Tumor diameter & $1.207(1.145-1.272)$ & 0 & $1.135(1.105-1.245)$ & 0.007 \\
\hline Liver capsule invasion & $2.371(1.436-3.918)$ & 0.001 & $1.242(0.701-2.241)$ & 0.458 \\
\hline Blood vessel invasion & $2.171(1.200-3.927)$ & 0.01 & $1.606(0.859-3.005)$ & 0.138 \\
\hline Differentiation & $2.234(1.535-3.253)$ & 0 & $1.701(1.116-2.594)$ & 0.014 \\
\hline BCLC staging & $2.598(1.872-3.605)$ & 0 & $1.224(0.720-2.081)$ & 0.454 \\
\hline incision margin & $0.957(0.625-1.466)$ & 0.841 & & \\
\hline Operative time & $1.002(1.000-1.005)$ & 0.048 & $1.000(0.998-1.003)$ & 0.762 \\
\hline Warm ischemia time & $0.994(0.974-1.015)$ & 0.592 & & \\
\hline Blood transfusion & $1.672(1.043-2.683)$ & 0.033 & $0.945(0.515-1.735)$ & 0.855 \\
\hline Preoperative TACE & $2.143(1.202-3.822)$ & 0.01 & $1.589(0.832-3.035)$ & 0.16 \\
\hline
\end{tabular}

$p=0.046$ ), and tumor differentiation (HR 1.487; 95\%CI $1.075-2.059 ; p=0.027)$.

Exploratory multivariate analysis using a stepwise Cox proportional-hazards model identified three variables as independent prognostic risk factors for survival (Table 5). These were preoperative AFP level (HR 1.767; 95\%CI $1.108-2.816 ; p=0.017$ ), number of tumor (HR 1.857; 95\% CI 1.071-3.221; $p=0.028$ ), tumor diameter (HR 1.135;
95\% CI 1.105-1.245; $p=0.007)$, and tumor differentiation (HR 1.701; 95\%CI 1.116-2.594; $p=0.014$ ).

\section{Discussion}

This study shows that preoperative serum AFP level is predictive of mortality after surgical resection independently of other prognostic factors. Although high 
AFP level increased risk of recurrence, it was not an independent negative prognosis factor for recurrence free survival by multivariate analysis. Increased preoperative serum AFP level has been reported by a large number of other studies to be an adverse prognostic factor both in early and advanced stages of HCC (Savastano et al., 1999; Ikai et al., 2004; Peng et al., 2004; Sugita et al., 2008). The finding of this study that AFP is independently predictive of poor survival is consistent with results of these studies.

In this study, we found that preoperative AFP levels in the female were higher than that in male patients. This finding was similar to some researchers (Zhou et al., 2013; Li et al., 2014). However, it is not unknown that the mechanism and clinical value of this finding.

Despite these promising results, the clinical use of AFP has been also shown to present some important limitations in sensitivity and specificity. In fact, low AFP levels have been described in HCC patients, while high levels might be detected in hepatic cirrhosis without HCC (Bertino et al., 2011). For instance, the level of AFP was increased in only $38 \%$ patients developing small HCC nodules (Sangiovanni et al., 2004). Indeed, AFP might be also useful in detecting other tumors (such as germ cell tumors of the ovary and testis and colorectal cancer) (Haibin et al., 2010; Nakagawa et al., 2012). The study by Giannini et al failed to show a prognostic value of AFP in well-compensated cirrhotic patients with single, small $\mathrm{HCC}(<3 \mathrm{~cm})$ treated with curative intent (Giannini et al., 2012). And, it's reported that AFP levels had no prognostic usefulness for female patients, probably because female patients usually had normal liver function, a lesser proportion of cirrhosis, and a greater proportion of single and small tumors, which may weaken the prognostic value of AFP (Li et al., 2014). Higher AFP level has been found to be associated with poorly differentiated tumors, biliary involvement, multifocal HCC, portal vein thrombosis, which themselves are known to be adverse prognostic factors in HCC (Nomura et al., 1989; Zhou et al., 2013). And it indicates that the prognosis value of preoperative AFP is affected by these factors to some extent. In our study, increased AFP exhibited characteristics of liver capsule invasion, low grade differentiation, and late BCLC stage. Thus, although currently recommended as a fundamental parameter for HCC screening in patients with cirrhosis, the prognostic capability of AFP in patients with HCC remains controversial and with important limitations. Our study shows that preoperative serum AFP is an independent adverse prognostic factor for mortality after surgical resection, independently of other prognostic factors including tumor size, tumor number, tumor grade differentiation, biliary or vascular involvement, and BCLC stage.

So far, it was not unclear that the exact mechanism of how AFP could be a prognostic indicator for HCC. Most researchers believe that AFP is released as a result of hepatocarcinogenesis, and is hence reflective of the proliferative activity and tumor burden. AFP may promote the proliferation of NIH 3T3 cells by binding its receptor to trigger the signal transduction pathway of cAMP-PKA and alter the expression of $\mathrm{K}$ - ras $\mathrm{p} 21$ gene (Li et al., 2002). AFP showed a capability to promote
HepG 2 cells by 3.5 folds, and the percentage of HepG2 cells in $\mathrm{S}$ phase was modestly increased after exposure to AFP (Li et al., 2011). AFP may play a regulatory role on angiogenesis and cell invasion during $\mathrm{HCC}$ development by increasing expression of vascular endothelial growth factor, vascular endothelial growth factor receptor 2, matrix metalloproteinases-2/9 (Meng et al., 2014). AFP may positively regulate cell proliferation by enhancing the apoptosis resistance via dysfunction of the p53/Bax/ cytochrome c/caspase-3 signaling pathway in AFPproducing HCC cell line (Yang et al., 2008). These findings suggest that AFP elevation might not only be just an epiphenomenon of malignant transformation, but may also actively participate in tumor proliferation.

The expression of AFP is regulated by many factors in different stages. Transcriptionally, AFP expression is regulated by a promoter region, a repressor region, and 3 enhancer regions. The promoter region is responsible for initiating AFP expression and binding of several transcription factors including hepatocyte nuclear factor-3 alpha (HNF-3alpha), hepatocyte nuclear factor-1 (HNF1), nuclear factor 1 (NF-1), and CAAT/enhancer binding protein (C/EBP) (Bois-Joyeux et al., 1994; Crowe et al., 1999). The repressor activity is modulated by binding of several transcription repressors such as zinc finger protein ZBTB20 and p53 represses AFP transcription. During carcinogenesis, various signaling pathways such as microRNA122/CUX1 deregulation and p53 mutation modulate the activity of these repressors, thereby alleviating AFP repression and AFP expression increasing (Kojima et al., 2011). Moreover, HBV viral protein, HBx, disrupts p53-mediated AFP repression through an association with DNA-bound p53, and large numbers of $\mathrm{HBV}$ integration into the host genome correlate with high serum AFP levels (Ogden et al., 2000; Sung et al., 2012). However, the detailed mechanisms of the AFP expression are still not completely understood.

In summary, preoperative serum AFP is a predictor of overall survival and recurrence-free survival independent of other clinicopathological factors among patients following surgical resection. The female patients had a higher preoperative AFP than male patients.

\section{Acknowledgements}

The study was supported by PUMC Youth Fund and the Fundamental Research Funds for the Central Universities (no. 33320140163).

\section{References}

Bertino G, Neri S, Bruno CM, et al (2011). Diagnostic and prognostic value of alpha-fetoprotein, des- $\gamma$-carboxy prothrombin and squamous cell carcinoma antigen immunoglobulin $\mathrm{M}$ complexes in hepatocellular carcinoma. Minerva Med, 102, 363-71.

Bois-Joyeux B, Danan JL (1994). Members of the CAAT/ enhancer binding protein, hepatocyte nuclear factor-1 and nuclear factor-1 families can differentially modulate the activities of the rat alpha fetoprotein promoter and enhancer. Biochem J, 301, 49-55.

Bosetti C, Levi F, Boffetta P, et al (2008). Trends in mortality 
Prognostic Significance of Preoperative Serum Alpha-fetoprotein in Hepatocellular Carcinoma in China

from hepatocellular carcinoma in Europe, 1980-2004. Hepatology, 48, 137-45.

Center MM, Jemal A (2011). International trends in liver cancer incidence rates. Cancer Epidemiol Biomarkers Prev, 20, 2362-8.

Chan SL, Chan AT, Yeo W (2009). Role of alpha-fetoprotein in hepatocellular carcinoma: prognostication, treatment monitoring or both? Future Oncol, 5, 889-99.

Crowe AJ, Sang L, Li KK, et al (1999). Hepatocyte nuclear factor 3 relieves chromatin-mediated repression of the alphafetoprotein gene. J Biol Chem, 274, 25113-20.

Debruyne EN, Delanghe JR (2008). Diagnosing and monitoring hepatocellular carcinoma with alpha-fetoprotein: new aspects and applications. Clin Chim Acta, 395, 19-26.

El-Serag HB (2011). Hepatocellular carcinoma. $N$ Engl J Med, 365, 1118-27.

Ferlay J, Shin HR, Bray F, et al (2010). Estimates of worldwide burden of cancer in 2008: GLOBOCAN 2008. Int J Cancer, 127, 2893-917.

Forner A, Vilana R, Ayuso C, et al (2008). Diagnosis of hepatic nodules $20 \mathrm{~mm}$ or smaller in cirrhosis: prospective validation of the noninvasive diagnostic criteria for hepatocellular carcinoma. Hepatology, 47, 97-104.

Giannini EG, Marenco S, Borgonovo G, et al (2012). Alphafetoprotein has no prognostic role in small hepatocellular carcinoma identified during surveillance in compensated cirrhosis. Hepatology, 56, 1371-9.

Gupta S, Bent S, Kohlwes J (2003). Test characteristics of alpha fetoprotein for detecting hepatocellular carcinoma in patients with hepatitis C. A systematic review and critical analysis. Ann Intern Med, 139, 46-50.

Haibin Z, Yue J, Yaxian X (2010). Primary yolk sac tumor of the omentum: a case report and literature review. Eur J Gynaecol Oncol, 31, 682-4.

Ikai I, Arii S, Kojiro M, et al (2004). Reevaluation of prognostic factors for survival after liver resection in patients with hepatocellular carcinoma in a Japanese nationwide survey. Cancer, 101, 796-802.

Jemal A, Bray F, Center MM, et al (2011). Global cancer statistics. CA Cancer J Clin, 61, 69-90.

Kim do Y, Han KH (2012). Epidemiology and surveillance of hepatocellular carcinoma. Liver Cancer, 1, 2-14.

Kojima K, Takata A, Vadnais C, et al (2011). MicroRNA122 is a key regulator of alpha fetoprotein expression and influences the aggressiveness of hepatocellular carcinoma. Nat Commun, 2, 338.

Lee SS, Shin HS, Kim HJ, et al (2012). Analysis of prognostic factors and 5-year survival rate in patients with hepatocellular carcinoma: a single-center experience. Korean J Hepatol, 18, 48-55.

Li MS, Li PF, Yang FY, et al (2002). The intracellular mechanism of alpha-fetoprotein promoting the proliferation of NIH 3T3 cells. Cell Res, 12, 151-6.

Li P, Wang SS, Liu H, et al (2011). Elevated serum alpha fetoprotein levels promote pathological progression of hepatocellular carcinoma. World J Gastroenterol, 17, 4563-71.

Li T, Qin LX, Gong X, et al (2014). Clinical characteristics, outcome, and risk factors for early and late intrahepatic recurrence of female patients after curative resection of hepatocellular carcinoma. Surgery, 156, 651-60.

Meng W, Li X, Bai Z, et al (2014). Silencing alpha-fetoprotein inhibits VEGF and MMP-2/9 production in human hepatocellular carcinoma cell. PLoS One, 9, 90660.

Nakagawa K, Koike S, Matsumura H, et al (2012). Alphafetoprotein producing rectal cancer. Gan To Kagaku Ryoho, 39, 671-4.
Nomura F, Ohnishi K, Tanabe Y (1989). Clinical features and prognosis of hepatocellular carcinoma with reference to serum alpha-fetoprotein levels. Analysis of 606 patients. Cancer, 64, 1700-7.

Ogden SK, Lee KC, Barton MC (2000). Hepatitis B viral transactivator $\mathrm{HBx}$ alleviates $\mathrm{p} 53$-mediated repression of alpha-fetoprotein gene expression. J Biol Chem, 275, 27806-14.

Peng SY, Chen WJ, Lai PL, et al (2004). High alpha-fetoprotein level correlates with high stage, early recurrence and poor prognosis of hepatocellular carcinoma: significance of hepatitis virus infection, age, $\mathrm{p} 53$ and beta-catenin mutations. Int J Cancer, 112, 44-50.

Sangiovanni A, Del Ninno E, Fasani P, et al (2004). Increased survival of cirrhotic patients with a hepatocellular carcinoma detected during surveillance. Gastroenterology, 126, 100514.

Savastano S, Miotto D, Casarrubea G, et al (1999). Transcatheter arterial chemoembolization for hepatocellular carcinoma in patients with Child's grade A or B cirrhosis: a multivariate analysis of prognostic factors. J Clin Gastroenterol, 28, 334-40.

Sugita S, Sasaki A, Iwaki K, et al (2008). Prognosis and postoperative lymphocyte count in patients with hepatocellular carcinoma who received intraoperative allogenic blood transfusion: a retrospective study. Eur $J$ Surg Oncol, 34, 339-45.

Sung WK, Zheng H, Li S, et al (2012). Genomewide survey of recurrent $\mathrm{HBV}$ integration in hepatocellular carcinoma. Nat Genet, 44, 765-9.

Tomasi TB Jr (1977). Structure and function of alpha-fetoprotein. Annu Rev Med, 28, 453-65.

Yang X, Zhang Y, Zhang L, et al (2008). Silencing alphafetoprotein expression induces growth arrest and apoptosis in human hepatocellular cancer cell. Cancer Lett, 271, 281-93.

Yu W, Rong W, Wang L et al (2014). R1 hepatectomy with exposure of tumor surface for centrally located hepatocellular carcinoma. World J Surg, 38, 1777-85.

Yuen MF, Tanaka Y, Fong DY, et al (2009). Independent risk factors and predictive score for the development of hepatocellular carcinoma in chronic hepatitis B.J Hepatol, 50, $80-8$.

Zhang XF, Qi X, Meng B, et al (2010). Prognosis evaluation in alpha-fetoprotein negative hepatocellular carcinoma after hepatectomy: comparison of five staging systems. Eur $J$ Surg Oncol, 36, 718-24.

Zhou J, Yan T, Bi X, et al (2013). Evaluation of seven different staging systems for alpha-fetoprotein expression in hepatocellular carcinoma after hepatectomy. Tumor Biol, 34, 1061-70. 